(2) Click for updates

Cite this: RSC Adv., 2014, 4, 61656

Received 1st October 2014 Accepted 6th November 2014

DOI: 10.1039/c4ra11601a

www.rsc.org/advances

\section{Dendritic polyglycerol cyclodextrin amphiphiles and their self-assembled architectures to transport hydrophobic guest molecules $\uparrow$}

\author{
Ana Campo Rodrigot. ${ }^{a}$ Shashwat Malhotrał, ${ }^{a}$ Christoph Böttcher, ${ }^{b}$ Mohsen Adeli*ac \\ and Rainer Haag*a
}

\begin{abstract}
Here we report for the first time a microwave assisted synthesis of polyglycerol dendron functionalized cyclodextrins (CD) with hydrophobic tails. These amphiphilic CDs consist of seven polyglycerol dendrons and fourteen alkyl chains on the primary and secondary rims of the cyclodextrin core, respectively. They self-assemble to form nanostructures in aqueous solutions and efficiently encapsulate hydrophobic aromatic guests. The size and shape of the selfassemblies and also their ability to encapsulate guest molecules depend on the generation of conjugated polyglycerol dendrons.
\end{abstract}

Well-defined nano-aggregates and nanostructures including micelles and vesicles have received extensive attention due to their versatile biomedical applications such as drug, dye, and gene delivery. They generally feature a core-shell structure made up of hydrophobic and hydrophilic blocks that facilitates drug/dye loading, and enhance their solubility for delivery purposes. $^{\mathbf{1 - 4}}$

Cyclodextrins (CDs) are macrocyclic oligosaccharides including $6(\alpha), 7(\beta)$, or $8(\gamma) \mathrm{D}-(+)$-glucopyranose units linked by $\alpha$ - $(1,4)$ glycosidic bonds. CDs have been widely used as host systems in supramolecular chemistry for fabricating various types of nanostructures because of their hydrophilic exterior surface as well as a hydrophobic interior cavity, which can accommodate a wide range of molecules as guests. ${ }^{5,6}$ However, in order to improve their physicochemical properties which in turn effect their biological applications, CDs have been modified with other types of macro-molecules. ${ }^{7,8}$ This approach has

\footnotetext{
anstitute for Chemistry and Biochemistry, Freie Universität Berlin, Takustr. 3, D-14195 Berlin, Germany. E-mail: haag@chemie.fu-berlin.de; Web: http://www. polytree.de; Fax: +49-30-838-53357; Tel: +49-30-838-52633

${ }^{b}$ Research Center of Electron Microscopy and the core facility "Biosupramol", Institute of Chemistry and Biochemistry, Freie Universität Berlin, Fabeckstrasse 36a, 14195 Berlin, Germany

'Department of Chemistry, Faculty of Science, Lorestan University, Khorramabad, Iran $\dagger$ Electronic supplementary information (ESI) available. See DOI: 10.1039/c4ra11601a

\$ These authors contributed equally to this study.
}

been widely used to produce nanostructures from cyclodextrin based amphiphiles in the recent years. ${ }^{\text {9-12 }}$ Although the resultant hybrid materials exhibit interesting properties induced by both macromolecules and CDs, they also exhibit long-term toxicity profiles as a result of their persistence inside the cells. $^{\mathbf{1 3 , 1 4}}$ Reproducible synthesis of well-defined systems is another main hurdle that must be met before polymer conjugated $\mathrm{CD}$ nanosystems can be considered for biomedical applications.

Amphiphilic, low molecular weight (LMW) dendron based architectures, in which a hydrophobic group at the focal point encourages LMW dendrons to self-assemble into a larger aggregate, were successfully synthesized and evaluated for their aggregation and other biological studies. ${ }^{15-18}$ Using polyglycerol (PG) dendrons, our research group has recently shown that such a strategy can enhance multivalent interactions for drug and gene delivery which leads to synergistic effects if both multifunctional dendrons and lipophilic tails are combined. ${ }^{\mathbf{1 9 - 2 3}}$

PG based dendrons with a tree-like structure and a large number of hydroxyl groups can serve as the hydrophilic part within an amphiphilic structure. Their water solubility and physiological safety, which is based on the very biocompatible glycerol building block, together with minimum non-specific interactions with protein, has stimulated researchers to use them for biomedical applications. ${ }^{\mathbf{2 4 - 2 7}}$ Furthermore, their post hydroxyl modifications offers excellent opportunities for multivalent interactions with biological substrates and has resulted in considerably stronger binding affinities compared to monovalent interactions. ${ }^{28}$ In addition, the degree of branching and thus flexibility can be varied by choosing a certain dendron generation. ${ }^{29,30}$

In the current study, we report on the design, synthesis and characterization of the well-defined cyclodextrin-polyglycerol dendron amphiphiles (CD-PG) and their self-assembly into different nanostructures (Fig. 1). Polyglycerol dendrons were synthesized using a divergent approach and conjugated to $\beta$-cyclodextrin ( $\beta$-CD) with fourteen hydrophobic tails by the convergent method to obtain amphiphilic CD-PGs dendrimers 


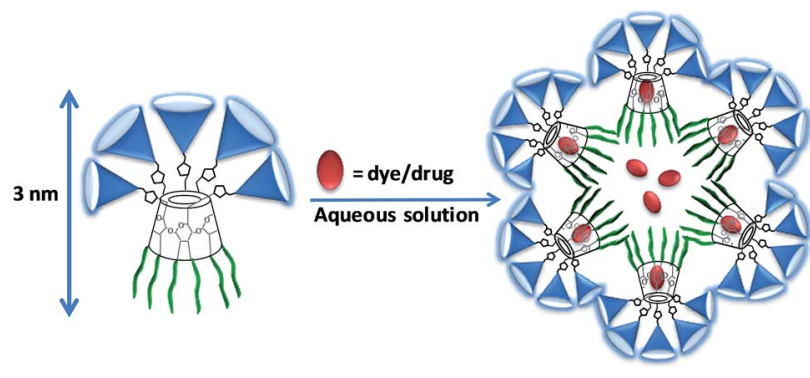

Fig. 1 Self-assembly of amphiphilic CD-PG dendrimers in aqueous solutions leads to nanostructures with two different domains to deliver hydrophobic guest molecules.

consisting of seven PG dendrons and fourteen aliphatic chains on the primary and secondary rims of cyclodextrin, respectively. Encapsulation of the hydrophobic guest molecules in different domains of their self-assembled architectures in aqueous solutions and toxicity of amphiphiles against A549 cell line was also investigated.

The synthesis of two amphiphilic CD-PG dendrimers, G1PG-CD and G2PG-CD which contain seven units of the first and second generations of polyglycerol dendrons on the primary side and fourteen $\mathrm{C}_{8}$-alkyl chains on the secondary side are presented in Scheme $\mathrm{S} 1 . \dagger$ The chemical structure of the synthesized G2PG-CD dendrimer is shown in Fig. 2.

The per-azido- $\beta$-cyclodextrin $\mathbf{4}$ was synthesized according to previously reported methods (Scheme S1†). ${ }^{31}$ The first- and second-generation propargyl functionalized PG dendrons, which were acetal protected in the case of G1 dendron 8 and deprotected in the case of G2 dendron 9, were synthesized according to our reported method for "click" dendrons. ${ }^{32,33}$ Treating octanoyl chloride with per-azido- $\beta$-CD, afforded alkylated per-azido- $\beta$-CD (Scheme S1 $\dagger$ ). Functionalization of the resulting compound on the primary rim with the first and second generations of PG dendrons was efficiently accomplished by employing a copper-catalyzed "click" reaction. This

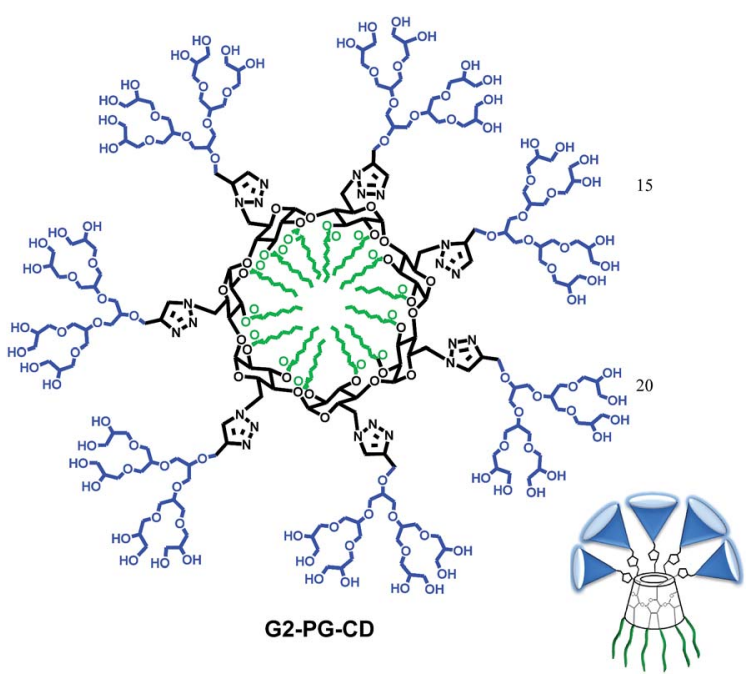

Fig. 2 Chemical structure of the amphiphilic G2PG-CD dendrimer. reaction was first carried out using $\mathrm{CuSO}_{4} \cdot 5 \mathrm{H}_{2} \mathrm{O}$-sodium ascorbate $(1: 1)$, DIPEA as base, in $1: 1$ mixture of binary solvents using conventional heating ranging from $80-160{ }^{\circ} \mathrm{C}$. Unfortunately none of the reaction conditions afforded a fully functionalized conjugate and only a mixture of partially functionalized conjugates was obtained.

A dramatic improvement in this key step was realized by using microwave irradiation. Interestingly, the 1,3-dipolar cycloaddition of alkylated per-azido- $\beta$-CD with the corresponding propargyl dendrons was efficiently carried out separately using $\mathrm{CuSO}_{4} \cdot 5 \mathrm{H}_{2} \mathrm{O}$-sodium ascorbate and 2,6-lutidine base in THF- $\mathrm{H}_{2} \mathrm{O}(1: 1)$ under microwave irradiation at $120^{\circ} \mathrm{C}$ for 30 min. After flash chromatography, acetal-protected form of G1PG-CD and the second generation amphiphilic conjugate G2PG-CD were obtained in $87 \%$ and $45 \%$ yields, respectively (Scheme $11^{\dagger}$ ). The acetal-protected form of G1PG-CD was then deprotected to afford the first generation cyclodextrin conjugate G1PG-CD in 92\% yield. Structures of G1PG-CD and G2PG-CD compounds were characterized by different spectroscopic methods, MALDI TOF and MS (see ESI, $\uparrow$ pages S6-S20).

Before investigating the ability of G1PG-CD and G2PG-CD compounds to encapsulate hydrophobic guest molecules, they were first evaluated in terms of their physicochemical properties, particularly regarding critical micelle concentration (CMC) and structure by fluorescence spectroscopy, dynamic light scattering (DLS) and cryo-transmission electron microscopy (cryo-TEM).

As it is explained in the ESI (Fig. S32 $\dagger$ ), 1,6-diphenyl-1,3,5hexatriene (DPH) was used as a probe to determine CMC of the amphiphiles. A wide range of concentration of amphiphile in the presence of a constant concentration of probe was evaluated. However, only one brake point in the fluorescence intensity was observed. Interestingly this gap was at concentration of $1 \mu \mathrm{M}$ in which all molecular probes were hosted by cyclodextrins. This result indicates that self-assembly has been occurred immediately after full complexation of guests (probe) molecules by the host molecules.

DLS experiments showed that G1PG-CD and G2PG-CD form self-assemblies with 44 and $19 \mathrm{~nm}$ sizes (by volume) in aqueous solutions, respectively (see Table S1 and Fig. S20-S21†). Since the hydrophilic block of G2PG-CD is bigger than that for G1PG$\mathrm{CD}$, it is able to form smaller aggregates. However, due to their size in both cases more complex aggregations are expected.

Cryo-TEM measurements revealed that G1PG-CD forms multilamellar vesicular structures in the diameter range of 10 to $400 \mathrm{~nm}$ and also in many cases shows a spot-like ultrastructure of the membrane (Fig. 3a). The dense association of seven G1 dendrons and seven triazole rings per molecule causes exceptionally high local electron density and allows for the localisation of molecular details in those areas where superposition of molecules occurs. Since the length of G1PG-CD is around 3 $\mathrm{nm}$ theoretically, membrane of vesicles with $6 \mathrm{~nm}$ thickness should be a bimolecular self-assembly. Interestingly, selfassembly of G2PG-CD leads to networks of mostly worm-like structures in aqueous solutions (Fig. 3b). High density dotlike entities most probably represent worms in an orientation more or less parallel to the electron beam, so that more density 


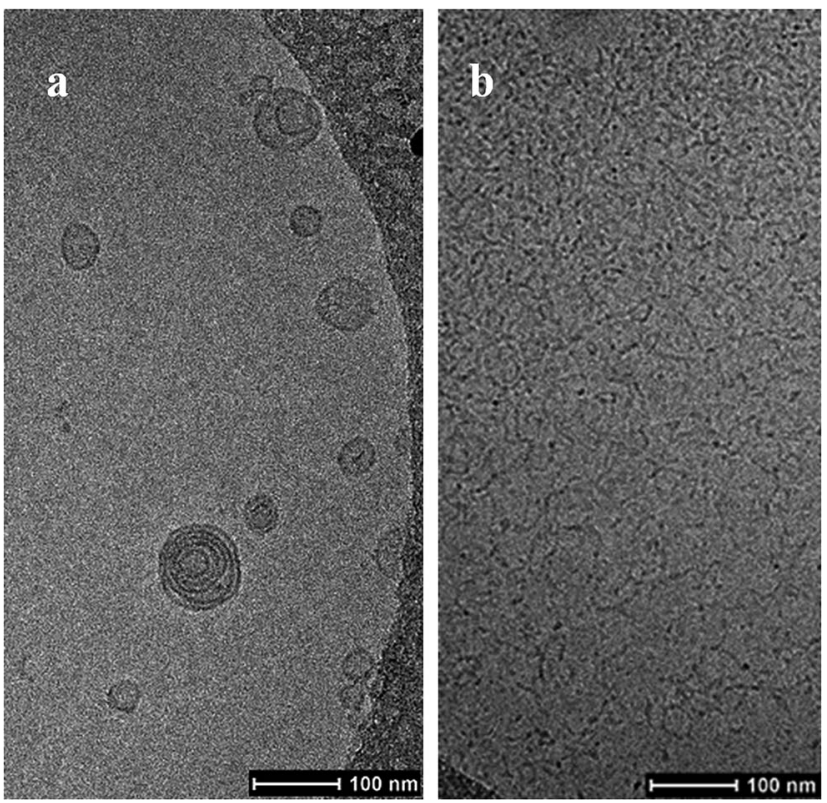

Fig. 3 Cryo-TEM images show that the shape and size of molecular self-assemblies of CD-PG dendrimers in aqueous solution depend on the volume of their hydrophilic PG head groups. G1PG-CD creates multilamellar vesicular structures (a), while G2PG-CD with bigger PG dendrons forms worm-like structures (b).

is added in the projection image of elongated assemblies. The diameter of the micelles is roughly in the order of 6-7 nm and corresponds also here to the dimension of the bimolecular assembly.

All the supramolecular structures clearly reflect the amphiphilic character of CD-PG perfect dendrimers by revealing structural details of their bimolecular assemblies, where the head to tail ratio obviously defines the curvature in the aggregates.

As expected from our earlier study the G1 derivatives tend to form less curved structures due to the significantly smaller head group volume if compared with G2 or even G3 analogs. ${ }^{19}$ Although single chained G1 amphiphiles assemble to form spherical and worm-like micelles, the formation of less curved vesicle membranes in the case of G1PG-CD can be explained by the combination of smaller $\mathrm{G} 1$ head groups and rigid cyclodextrin platforms with many tails. The G2PG-CD derivative with the larger head group, however, tends to form assemblies with a higher curvature. Also here the ultrastructure is different compared to the single chained G2-derivatives, which exclusively form spherical micelles. ${ }^{19}$ Again, the cyclodextrin platform adds a grain of more rigidity and therefore rather elongated cylindrical micelles than spherical micelles are observed for G2PG-CD.

Self-assembly of amphiphilic CD-PG dendrimers toward well-defined vesicles in aqueous solutions and also presence of a well-known host molecule, cyclodextrin, in their core structure stimulated us to investigate their host-guest properties by using the drug nimodipine and the dye nile red as neutral hydrophobic guests. Addition of the guests into an aqueous solution of the hosts, G1PG-CD and G2PG-CD, led to clear homogeneous solutions upon stirring. Each of the guest molecules were incorporated separately in both host molecules by the solid uptake method. After encapsulation, the loading of the guests in hosts was determined by using UV-vis spectroscopy. As shown in Table S2, $\uparrow$ the loading mole percentage for nimodipine and nile red determined by UV-vis spectroscopy was found to be 85 and $100 \mathrm{~mol} \%$ in the host G1PG-CD, respectively, while in the host G2PG-CD lower loadings of 50 and $15 \mathrm{~mol} \%$ have been observed respectively. To note, in comparison to the encapsulation capability of our previously reported dendritic amphiphiles,,$^{34}$ the transport behaviour of these new systems is highly efficient. This could well be attributed to an additional presence of the encapsulation capabilities of one of the host i.e. cyclodextrin, present in current novel architectures G1PG-CD and G2PG-CD. The corresponding UV-vis graphs are shown in the ESI as Fig. S26-S30. $\dagger$ Table S2 $\uparrow$ shows that each mole of G1PG-CD included in self-assemblies, encapsulate 0.85 and 1 eq. of nimodipine and nile red, respectively. Therefore in the case of nile red and almost for nimodipine, complexation is $1: 1$. This type of inclusion complex between nile red or nimodipine and $\beta$-CD $(1: 1)$ has been reported previously. ${ }^{35}$ Since cyclodextrin is more effective than the alkyl chains in the competitive interaction with the guest molecules mentioned above ${ }^{36}$ this result show that all cyclodextrin cavities are often occupied with the hydrophobic guests. In this case alkyl chains interact together to create self-assemblies mainly and attend in encapsulation process minimally. DLS diagrams for G1PG-CDNimo and G1PG-CD-NR (G1PG-CD assemblies containing encapsulated nimodipine and nile red respectively) showed a small, close to the pristine self-assembly, and a size of several hundred nanometres (Table S1 and Fig. S22-S23†).

As long as guest molecules remain inside the cyclodextrin cavities, size of self-assemblies change slightly and are quite close to the pristine analogs. However, due to the dynamic nature of inclusion complexes and as a result of the mentioned competitive interaction, guest molecules are probably able to locate between the alkyl chains as well. Since interactions between alkyl chains are the main driving force to keep the shape and size of self-assemblies, interfering in these interactions by guest molecules will affect their sizes dramatically.

Encapsulation efficiency of G2PG-CD is somewhat lower (Table S2 $\dagger$ ), because bulky PG groups hamper interactions between the cavity of cyclodextrin and guest molecule as it has been reported in similar cases. ${ }^{36}$ Also, the self-assemblies of G2PG-CD are smaller than that of G1PG-CD in aqueous solutions, that could also be the reason for its low encapsulation efficiency. DLS experiments show that the size of selfassemblies of G2PG-CD do not change as much as in the case of G1PG-CD upon encapsulation of guest molecules.

In order to get a preliminary assessment of the cytotoxicity, we performed MTT assays on A549 cells treated with G1PG-CD and G2PG-CD. As revealed in Fig. S31, $\dagger$ G1PG-CD and G2PGCD both showed similar cell viability profiles and is only observed cellular toxicity above $5 \mu \mathrm{M}$.

In conclusion, we have successfully synthesized amphiphilic dendritic CDs that consist of seven polyglycerol dendrons and fourteen alkyl chains conjugated to a $\beta$-cyclodextrin core 
through microwave assisted click chemistry. The synthesized amphiphiles self-assembled and formed defined nanostructures in aqueous solutions. Encapsulation efficiency, size and shape of nanostructures can be regulated by using different generations of PG dendrons. Vesicle-like self-assemblies with bigger sizes and higher encapsulation efficiency in which guest molecules settle down in the cavity of cyclodextrin mainly, can be obtain by G1 dendrons. On the other hand, worm-like aggregates with smaller sizes and lower encapsulation efficiency are achieved with more bulky G2 dendrons. These motifs are an important step towards the development of efficient, nontoxic, fully functionalized, and readily tailorable amphiphiles with discrete molecular weights for drug delivery.

\section{Acknowledgements}

We thank the BMBF (IGCST) and the DFG (SFB 765) as well as the core facility "Biosupramol" for financial support, as well as Dr Stefanie Wedepohl for the cytotoxicity studies.

\section{Notes and references}

1 M. E. Davis and M. E. Brewster, Nat. Rev., 2004, 3, 1023-1035. 2 J. Zhou and H. Ritter, Polym. Chem., 2010, 1, 1552-1559.

3 P. Jansook, S. V. Kurkov and T. Loftsson, J. Pharm. Sci., 2010, 99, 719-729.

4 V. Villari, A. Mazzaglia, R. Darcy, C. M. O'Driscoll and N. Micali, Biomacromolecules, 2013, 14, 811-817.

5 A. Harada, Y. Takashima and H. Yamaguchi, Chem. Soc. Rev., 2009, 38, 875-882.

6 M. E. Brewster and T. Loftsson, Adv. Drug Delivery Rev., 2007, 59, 645-666.

7 A. Poorjavadi, M. Yazdi and M. Adeli, New J. Chem., 2013, 37, 295.

8 D. Duchêne, G. Ponchel and D. Wouessidjewe, Adv. Drug Delivery Rev., 1999, 36, 29-40.

9 M. M. Becker, Z. Zeng and B. J. Ravoo, Eur. J. Org. Chem., 2013, 30, 6831-7684.

10 U. Kauscher, M. C. A. Stuart, P. Drücker, H. J. Galla and B. J. Ravoo, Langmuir, 2013, 29, 7377-7383.

11 R. Auzely-Velty, F. Djedaïni-Pilard, S. Desert, B. Perly and Th. Zemb, Langmuir, 2000, 16, 3727-3734.

12 M. Adeli, F. Abedi, A. K. Fard, B. K. Chegeni and F. Bani, Nanomedicine, 2013, 9, 1203-1213.

13 O. Jazkewitsch, A. Mondrzyk, R. Staffel and H. Ritter, Macromolecules, 2011, 44, 1365-1371.

14 S. C. Chan, S. W. Kuo and F. C. Chang, Macromolecules, 2005, 38, 3099-3107.

15 I. Toth, T. Sakthivel, A. F. Wilderspin, H. Bayele, M. O'Donnell, D. J. Perry, K. J. Pasi, C. A. Lee and A. T. Florence, STP Pharma Sci., 1999, 9, 93-99.

16 K. Ewert, A. Ahmad, H. M. Evans, H.-W. Schmidt and C. R. Safinya, J. Med. Chem., 2002, 45, 5023-5029.
17 D. Joester, M. Losson, R. Pugin, H. Heinzelmann, E. Walter, H. P. Merkle and F. Diederich, Angew. Chem., Int. Ed., 2003, 42, 1486-1490.

18 S. P. Jones, N. P. Gabrielson, D. W. Pack and D. K. Smith, Chem. Commun., 2008, 4700-4702.

19 B. Trappmann, K. Ludwig, M. R. Radowski, A. Shukla, A. Mohr, H. Rehage, C. Böttcher and R. Haag, J. Am. Chem. Soc., 2010, 132, 11119-11124.

20 A. Richter, A. Wiedekind, M. Krause, T. Kissel, R. Haag and C. Olbrich, Eur. J. Pharm. Sci., 2010, 40, 48-55.

21 R. Tyagi, S. Malhotra, A. Sedighi, M. Weber, A. Schäfer, A. Thunemann and R. Haag, J. Phys. Chem. C, 2013, 117, 12307-12317.

22 S. Malhotra, H. Bauer, A. Tschiche, A. Staedtler, A. Mohr, M. Calderon, V. Parmar, L. Hoeke, S. Sharbati, R. Einspanier and R. Haag, Biomacromolecules, 2012, 13, 3087-3098.

23 A. Tschiche, A. M. Staedtler, S. Malhotra, H. Bauer, C. Böttcher, S. Sharbati, M. Calderón, M. Koch, T. M. Zollner, A. Barnard, D. K. Smith, R. Einspanier, N. Schmidt and R. Haag, J. Mater. Chem. B, 2014, 2, 2153.

24 M. Calderón, M. A. Quadir, S. K. Sharma and R. Haag, Adv. Mater., 2010, 22, 190-218.

25 W. Tao, Y. Liu, B. Jiang, S. Yu, W. Huang, Y. Zhou and D. Yan, J. Am. Chem. Soc., 2012, 134, 762-764.

26 M. Adeli, A. Kakanejadifard, M. Khani, F. Bani, R. Kabiri and M. Sadeghizad, J. Mater. Chem. B, 2014, 2, 3589.

27 M. Martinelli, M. Calderon, C. I. Alvarez and M. C. Strumia, React. Funct. Polym., 2007, 67, 1018-1026.

28 C. Fasting, C. A. Schalley, M. Weber, O. Seitz, S. Hecht, B. Koksch, J. Dernedde, C. Graf, E.-W. Knapp and R. Haag, Angew. Chem., Int. Ed., 2012, 51, 10472.

29 R. Haag and F. Kratz, Angew. Chem., Int. Ed., 2006, 45, 11981215.

30 A. Benkhaled, H. Cheradame, O. Fichet, D. Teyssié, W. Buchmann and P. Guégan, Carbohydr. Polym., 2008, 73, 482-489.

31 B. I. Gorin, R. J. Riopelle and G. R. J. Thatcher, Tetrahedron Lett., 1996, 37, 4647-4650.

32 S. Malhotra, M. Calderon, A. K. Prasad, V. S. Parmar and R. Haag, Org. Biomol. Chem., 2010, 8, 2228-2237.

33 S. Gupta, B. Schade, S. Kumar, C. Boettcher, S. K. Sharma and R. Haag, Small, 2013, 9, 894-904.

34 C. S. Popeney, M. C. Lukowiak, C. Böttcher, B. Schade, P. Welker, D. Mangoldt, G. Gunkel, Z. Guan and R. Haag, ACS Macro Lett., 2012, 1, 564-567; X. Yang, W. Ke, P. Zi, F. Liu and L. Yu, J. Pharm. Sci., 2008, 97, 2702-2719.

35 B. Jana, S. Ghosh and N. Chattopadhyay, J. Photochem. Photobiol., B, 2013, 126, 1-10.

36 F. Kopecký, B. Kopecká and P. Kaclík, J. Inclusion Phenom. Macrocyclic Chem., 2001, 39, 215-217. 